\title{
Application of Dual Beam FIB to the metrology of nanostructured photovoltaic devices
}

\author{
G. McMahon, ${ }^{*} \mathrm{~J}_{\text {. Rybczynski, }}{ }^{+}$Y. Wang, ${ }^{+}$Y. Gao, ${ }^{*}$ N. Argenti, ${ }^{+}$K. Kempa, ${ }^{*}$, Z.F. Ren, ${ }^{*,}$ and M.J. \\ Naughton ${ }^{*},+$
}

* Department of Physics, Boston College, Chestnut Hill, MA 02467

+ Solasta Inc., Newton, MA 02458

Today's solar cell technologies are generally a compromise of their optical and electronic properties. They must be thick enough to effectively absorb sunlight, yet sufficiently thin to efficiently extract the generated charge. Increasing the efficiency of solar cells while maintaining reasonable production costs are prerequisites for widespread consumer acceptance of this renewable energy alternative to fossil fuels.

A novel solar cell architecture based on a dense array of nanostructures [1] that holds several advantages over the conventional planar configuration has recently been developed [2]. Light is collected by nanoantennae and channeled into the photovoltaic material [2]. Generated charge carriers then diffuse short (nanoscale) distances to be extracted. The Solasta architecture effectively separates the photon and charge transport directions.

This novel design has led to several metrology challenges: Measurement of layer thicknesses at the top, base and sides of the nanostructures and determining the conformity of coatings would be challenging and time consuming by conventional cross-sectional methods. We illustrate with several examples how we have largely eradicated these issues by application of dual beam FIB (JEOL Multibeam 4500) methods.

For routine cross-sectional analyses, we use the standard methodology of rough cutting an approximately $15 \mu \mathrm{m} \times 15 \mu \mathrm{m}$ box using a $30 \mathrm{kV} \mathrm{Ga}^{+}$beam current of $1 \mathrm{nA}$ and dose of $8 \mathrm{nC} / \mu^{2}$, and follow by polishing the cross-sectional face at a beam current of $100 \mathrm{pA}$. Depending upon the sample, we may first deposit a $\mathrm{C}$ protective surface coating to preserve the top surface during milling, but have found that depending upon the nature of the sample this is sometimes not possible, yet useful results are nonetheless obtained in its absence. We present SEM images $\left(5 \mathrm{kV}, \mathrm{LaB}_{6}\right.$ emitter) of a typical longitudinal section through a portion of one of our nano-architectures with a FIB-deposited $\mathrm{C}$ protective layer in Fig. 1. We can rapidly determine layer conformity and thicknesses at the top, bottom and sides of the structures.

Yet this only yields a small snapshot of the total structure. To ensure that such images are typical of the entire solar cell array, we make use of the serial sectioning and imaging capability of the dual beam FIB. We use the ion beam to progressively slice into the cross-sectional face at 40-60 nm intervals and automatically image the new exposed face using the electron beam. The result is a stack of 200-400 images through 10-30 rows of nanostructures which can be converted to a video sequence or used to create a volume rendering of the analyzed region as shown in Fig. 2. 
We may also use the FIB to cross-section the structures as shown in Figure 3a. By using a field emission SEM (JEOL 7001F) at low $\mathrm{kV}$ accelerating voltage, we can use EDS to map the chemical makeup of the individual layers (Figure $3 b$ ).

\section{References}

[1] J. Rybczynski et al., Appl. Phys. Lett. 90 (2007) 021104

[2] Solasta Inc., Newton, MA (to be published)

[3] K. Kempa et al., Appl. Phys. Lett. 92 (2008) 043114
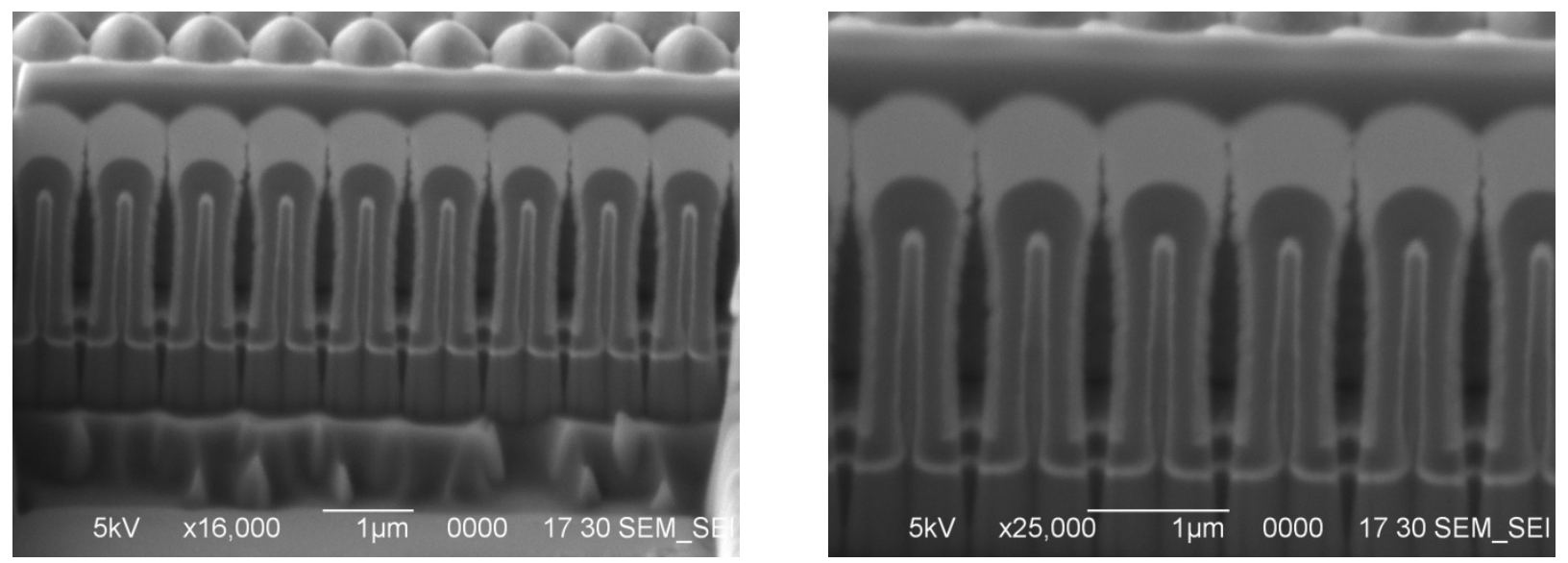

FIG 1: SEM secondary electron images of FIB prepared longitudinal section through nanostructured solar cell array.

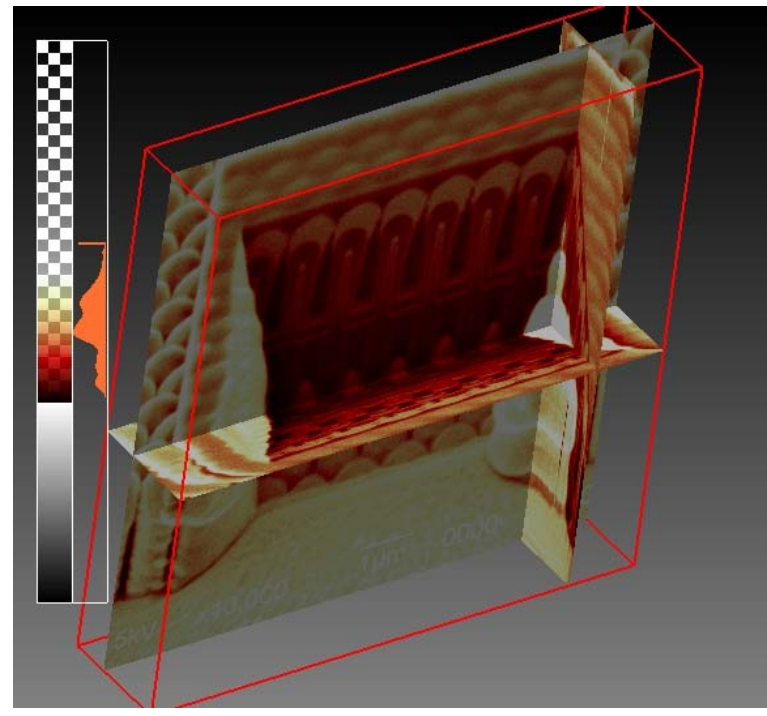

FIG. 2: 3-D representation of nanostructures obtained by automated FIB, sequentially slicing and SEM imaging.
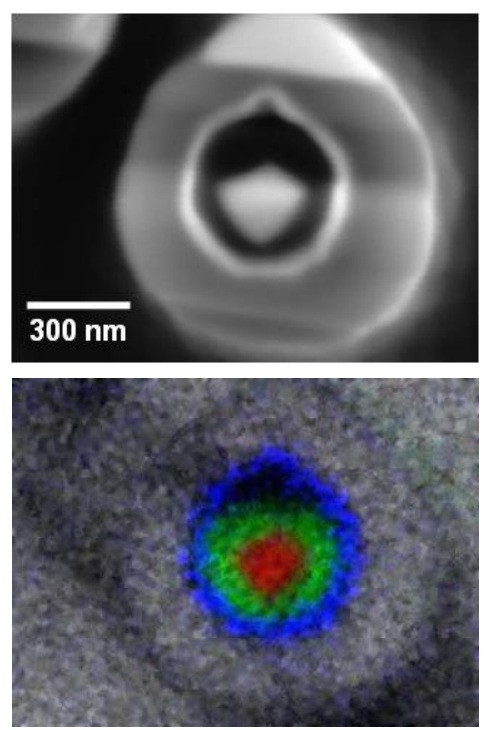

FIG. 3: (Top) Field emission SEM image of FIB cross-sectioned nanostructure;

(Bottom) Corresponding EDS X-ray map (elements proprietary). 